\title{
Update on the Cardiovascular Benefits of Sodium-Glucose Co-Transporter-2 Inhibitors: Mechanism of Action, Available Agents and Comprehensive Review of Literature
}

\author{
Randa Abdelmasiha, ${ }^{\mathrm{a}, \mathrm{b}}$, Ramy Abdelmaseih ${ }^{\mathrm{a}, \mathrm{b}, \mathrm{e}} \mathbb{C}$, Ravi Thakker ${ }^{\mathrm{c}}$, Mohammed Faluk ${ }^{\mathrm{a}, \mathrm{b}}$, \\ Arroj Ali ${ }^{a}$, , M. Mrhaf Alsamman ${ }^{a, b}$, Syed Mustajab Hasanª
}

\begin{abstract}
Despite the currently established treatment for heart failure (HF), HF remains a growing public healthcare problem with an increasing burden. Therefore, novel therapeutic innovations are needed to overcome this issue and improve HF prognosis. Sodium-glucose co-transporter-2 inhibitors (SGLT2i) are state-of-the-art in type 2 diabetes mellitus management. They inhibit the reabsorption of glucose from the proximal renal tubules, leading to increased glycosuria and decreased plasma glucose levels. SGLT2 $i$ use is growing significantly, especially after recent clinical trials demonstrating favorable cardiovascular and renal protective effects independently of blood glucose-lowering. The mechanisms by which SGLT2i demonstrate their cardio-renal protective effects remain incompletely understood but are thought to be related to potential diuretic and natriuretic effects along with other mechanisms that will be discussed in this article. Over the past few years, there has been significant research on the safety, efficacy, and quality of this class of medications. Here, we review the current guideline-directed medical therapy for HF, focus on SGLT2i mechanism of action and potential role in HF patients, and finally summarize the cardiovascular clinical trials with SGLT2.
\end{abstract}

Keywords: Sodium-glucose cotransporter-2 inhibitors; Heart failure with reduced ejection fraction; Heart failure with preserved ejection fraction; Cardiovascular disease; Canagliflozin; Dapagliflozin; Empagliflozin; Ertugliflozin

Manuscript submitted April 11, 2021, accepted April 26, 2021

Published online June 5, 2021

aUniversity of Central Florida College of Medicine, Graduate Medical Education, Orlando, FL, USA

${ }^{b}$ Ocala Regional Medical Center, Internal Medicine Residency Program, Ocala, FL, USA

cUniversity of Texas Medical Branch, Internal Medicine Residency Program, Galveston, TX, USA

dNorth Florida Regional Medical Center, Internal Medicine Residency Program, Gainesville, FL, USA

${ }^{\mathrm{e}}$ Corresponding Author: Ramy Abdelmaseih, University of Central Florida, Orlando, FL, USA. Email: ramy.abdelmaseih@hcahealthcare.com

doi: https://doi.org/10.14740/cr1268

\section{Introduction}

Despite the enormous improvement in cardiovascular care and the emergence of modern therapeutic innovations over the past several decades, heart failure (HF) continues to represent a nationwide healthcare problem accounting for $8.5 \%$ of cardiovascular-related deaths, which remains the number one leading cause of all deaths worldwide, and for about $13.4 \%$ of total United States death causes [1]. In the United States, about 380,000 deaths per year are caused by HF, with an estimated rate of one in every eight deaths. Currently, about 6.2 million adults are diagnosed with HF in the United States. The incidence of HF increases with age, with a reported annual incidence of 12 per 1,000 population in patients aged 65 years and older, and lifetime likelihood of developing HF of about $20 \%$ at all ages above 40 years [2].

HF is considered the primary hospitalization cause in the elderly population, with re-hospitalization rates exceeding $50 \%$ during the 6 months following discharge [3] and 5-year mortality rates approaching $50 \%$, increasing the burden on the healthcare system in the United States with an estimate of $\$ 43.6$ in 2020 with an estimated total rise of about $127 \%$ to $\$ 69.7$ billion by 2030 [4].

$\mathrm{HF}$ is a pathophysiologic state that occurs in patients with inherited or acquired cardiac structure/function abnormalities that result in failure in pumping blood at an optimal rate matching the tissue metabolism, leading to the development of a constellation of clinical symptoms (dyspnea, fatigue, or chest pain) and signs (edema, rales, S3 gallop, jagular venous distention, or hepatomegaly) [5]. HF can be classified using the New York Heart Association (NYHA) scale or the American College of Cardiology/American Heart Association (ACC/AHA) staging system into four classes or four stages, respectively [6, 7]. Diagnosis is mainly clinical and can be aided by using the Framingham criteria [5]. HF can also be categorized depending on the ejection fraction (EF) into preserved $\mathrm{EF}(\mathrm{EF}>50 \%$; HFpEF), midrange EF (EF between $40 \%$ and $49 \%$; HFmrEF), and reduced $\mathrm{EF}(\mathrm{EF}<40 \%$; HFrEF). Recent trends indicate that HFpEF prevalence is increasing from $41 \%$ to $56.2 \%$ of cases, relatively to $\mathrm{HFrEF}$, likely due to lower prevalence of 
coronary artery disease (CAD) and rising hypertension rates among HF patients [8].

$\mathrm{HF}$ risk factors include CAD, cigarette smoking, hypertension, obesity, diabetes, arrhythmias, infections, congenital heart diseases, valvular heart disease, drug use, idiopathic cardiomyopathy, infiltrative diseases, anemia, thyroid disorders, and nutritional deficiencies.

Management of HF depends on the severity and the acuity of the presentation. Non-pharmacologic therapies include oxygen supply, pain control, fluid restriction, daily weight measurement, and physical activity. Pharmacologic therapies include optimal control of comorbidities including hypertension and arrhythmias, diuretics, beta-blockers, angiotensinconverting enzyme inhibitors (ACEi), or angiotensin II receptor blockers (ARBs), angiotensin receptor-neprilysin inhibitor (ARNI), vasodilators and inotropic agents, digoxin, ivabradine, soluble guanylate cyclase stimulators, and anticoagulants. Despite the marked improvement in medical and device therapy, HF mortality following hospitalization is $10.4 \%$ at 30 days, $22 \%$ at 1 year, and exceeding $50 \%$ at 5 years [9]. Hence, the crucial need for HF exacerbation prevention in individuals with risk factors is through optimal control of their comorbidities, as well as reduction of HF re-hospitalization rates. Here, we shed light on the role of sodium-glucose co-transporter-2 inhibitors (SGLT2i) in HF patients and their impact on cardiovascular outcomes.

\section{SGLT2i Mechanisms}

SGLT2i, the novel class of oral antidiabetic agents, are currently recommended as second-line drugs for type 2 diabetes mellitus (T2DM). Though, they can be used as first-line drugs [10], and also, off-label, for type 1 diabetes [11]. The first SGLT2 $\mathrm{i}$ to be FDA-approved was canagliflozin (invokana, followed by dapagliflozin $\left(\right.$ Farxiga $^{\circledR}$ ), then empagliflozin (Jardiance $^{\circledR}$ ), and ertugliflozin (Steglatro $\left.{ }^{\circledR}\right)$ ). SGLT2 $i$ use is growing significantly, due to their unique mechanism of action, and their favorable cardiovascular and renal outcomes, independent of glycemic control, that have been established in several large, placebo-controlled clinical trials.

SGLT2 $i$ act independently of insulin level, by blocking the SGLT2 protein responsible for glucose reabsorption in the proximal renal tubule, promoting glycosuria and thereby modestly lowering elevated blood glucose levels in patients with T2DM. Moreover, SGLT2i have pro-diuretic effects; though the mechanism of action on a cellular level is not well explained, it has been linked to the filtered load of glucose leading to synergistic effects if combined with other diuretics. SGLT2i can also cause natriuresis, at least in the early phase of treatment, by inhibiting the transport of sodium for every molecule of unabsorbed glucose, resulting in systemic reninangiotensin-aldosterone (RAS) activation as a compensatory mechanism $[12,13]$. Other suggested mechanisms include attenuation of cardiac inflammation and fibrosisoxidative stress reduction, arterial stiffness reduction, improved endothelial function, reductions in blood pressure, and other renal protective effects. These mechanisms lead to cardiovascular benefits through reduction in left ventricular (LV) preload and afterload, leading to improved systolic and diastolic functions and reduction in LV mass. Also, it can potentially lead to improved myocardial energy production in the form of cardiac ketones and increased cardiac output, heart rate, oxygen consumption, and coronary flow through increased glucagon levels [14, 15].

SGLT2 $i$ use has grown significantly, especially after recent clinical trials that demonstrated their favorable cardiorenal protective effects, with impressive reductions in $\mathrm{HF}$ hospitalization, slow progression of kidney disease, reduced major adverse cardiovascular events (MACEs) and deaths. In patients with T2DM and HF, meta-analyses of the three major cardiovascular outcome trials (CVOTs) of canagliflozin, dapagliflozin, and empagliflozin with 34,322 patients have shown salutary effects. SGLT2i compared to placebo have reduced MACE (86.9 vs. 99.6 events/1,000 patient-years, hazard ratio (HR): $0.89,95 \%$ confidence interval $(\mathrm{CI}): 0.83-0.96 ; \mathrm{P}=$ $0.0014)$ with benefit seen in patients who already have established atherosclerotic cardiovascular disease (ASCVD) (HR: $0.86,95 \%$ CI: $0.8-0.93 ; \mathrm{P}=0.0501$ ), and a composite outcome of cardiovascular death or hospitalization for HF (48.2 vs. 65.6 events/1,000 patient-years, HR: $0.77,95 \% \mathrm{CI}: 0.71$ - 0.84; P $<0.0001$ ), regardless of the presence of ASCVD or $\mathrm{HF}$ at baseline [16]. Moreover, the same meta-analyses have shown that SGLT2 $i$ decreased the progression of renal disease by $45 \%$ (HR: $0.55,95 \% \mathrm{CI}: 0.48-0.64$; P $<0.0001$ ), regardless of ASCVD. The extent of SGLT2i benefits varied with variations in renal function baseline, with a bigger reduction in HF hospitalization $(\mathrm{P}=0.0073)$ and lesser reduction in renal disease progression $(\mathrm{P}=0.0258)$ in patients with more severe baseline renal disease. Other observational studies reported similar results $[17,18]$.

\section{CVOTs}

\section{EMPA-REG OUTCOME}

The EMPA-REG OUTCOME trial (Empagliflozin, Cardiovascular Outcomes, and Mortality in Type 2 Diabetes) published in 2015 [19] was conducted among patients ( $\geq 18$ years) with T2DM at high risk for cardiovascular events to investigate whether administering a daily dose of empagliflozin 10 or 25 $\mathrm{mg}$ reduces the cardiovascular mortality, nonfatal myocardial infarction (MI), and nonfatal strokes in comparison to placebo. A total of 7,020 patients were randomized to a daily dose of empagliflozin $10 \mathrm{mg}(\mathrm{n}=2,345)$, a daily dose of empagliflozin $25 \mathrm{mg}(\mathrm{n}=2,342)$ and placebo $(\mathrm{n}=2,333)$. The primary outcome was a composite of cardiovascular death, nonfatal MI (excluding silent MI), or nonfatal strokes. The empagliflozin group had a significant reduction in the primary outcome compared to the placebo group (10.5\% vs. $12.1 \%$, HR: $0.86,95 \%$ CI: $0.74-0.99 ; \mathrm{P}=0.04$ for superiority and $\mathrm{P}<0.001$ for noninferiority). The secondary outcome consisted of the primary outcome and unstable angina hospitalization. No significant difference has been reported (12.8\% vs. $14.3 \%$, HR: $0.89,95 \%$ CI: $0.78-1.01 ; \mathrm{P}=0.08$ for superiority and $\mathrm{P}<0.001$ for noninferiority). As compared to the placebo group, empagliflozin 
resulted in significantly lower risk of cardiovascular death, any cause death, and HF hospitalization. Adverse events that were more common in the empagliflozin arm include genital infections, and urosepsis. The study concluded that empagliflozin led to lower rates of the primary outcome, and any cause death compared to placebo. However, the study was criticized because each individual empagliflozin arm did not reach statistical significance in outcomes.

\section{CANVAS}

The CANVAS trial (canagliflozin and cardiovascular and renal events in type 2 diabetes) published in 2017 [20] was conducted among patients ( $>30$ years) with T2DM at high risk for cardiovascular events to investigate whether administering a daily dose of canagliflozin 100 or $300 \mathrm{mg}$ reduces cardiovascular mortality, nonfatal MI, or nonfatal strokes. A total of 10,142 patients were randomized in this trial with $96 \%$ completion rate. The primary outcome was a composite cardiovascular deaths, nonfatal MI, or nonfatal strokes. Less patients in the canagliflozin arm than in the placebo group had a primary outcome event (26.9\% vs. $31.5 \%$ events/1,000 patient-years, HR: $0.86,95 \%$ CI: $0.75-0.97 ; \mathrm{P}=0.02$ for superiority and $\mathrm{P}$ $<0.001$ for non-inferiority). The secondary outcomes were: all-cause mortality, cardiovascular mortality, progression of albuminuria (defined as $>30 \%$ increase in albuminuria and a change from either normoalbuminuria to microalbuminuria or macroalbuminuria, or from microalbuminuria to macroalbuminuria), $40 \%$ reduction in estimated glomerular filtration rate (eGFR), renal replacement therapy or death from renal causes, any-cause hospitalization, HF hospitalization, and cardiovascular mortality and hospitalization for HF. No significant difference in all-cause mortality or cardiovascular mortality was reported. Also, no significant difference in effects for primary outcome, or exploratory cardiovascular outcomes was reported between the CANVAS and CANVAS-R groups. As compared to the placebo group, canagliflozin resulted in significantly lower rates of progression of albuminuria, with more reduction in the CANVAS-R group compared to the CANVAS group. Also, regression of albuminuria was more frequently reported among the canagliflozin. The composite outcome of sustained $40 \%$ reduction in eGFR, renal replacement therapy or renal death occurred less frequently among the canagliflozin group (5.5\% vs. $9 \%$ events/1,000 patient-years, HR: $0.6,95 \%$ CI: 0.47 - 0.77) without significant outcome seen between CANVAS and CANVAS-R groups. Any-cause hospitalization was less frequent in the canagliflozin group but statistically insignificant ( $118.7 \%$ vs. $131.1 \%$ events/1,000 patient-years, HR: 0.94, 95\% CI: 0.88 - 1.00). However, HF hospitalization and a composite of cardiovascular mortality and HF hospitalization were significantly lower in the canagliflozin group. Adverse events included genital infections, increased rate of amputation, and increased bone fracture (statistically insignificant). The study concluded that canagliflozin led to a lower risk of cardiovascular events, but a greater risk of amputation, primarily at the level of the toe or metatarsal. However, the study was criticized because of the few participants with baseline chronic kidney disease (CKD) and the few end-stage renal disease (ESRD) events.

\section{CREDENCE}

The CREDENCE trial published in 2019 [21] was conducted among patients ( $>30$ years) with T2DM and diabetic nephropathy treated with renin-angiotensin system blockade to investigate whether administering a daily dose of canagliflozin 100 mg reduces the risk of ESRD, serum creatinine baseline doubling, and renal or cardiovascular deaths compared to placebo. A total of 4,401 patients were randomized to canagliflozin (n $=2,202)$, and placebo $(n=2,199)$. The primary outcome was a composite of ESRD, serum creatinine baseline doubling, or renal and cardiovascular deaths. The primary outcome was significantly lower in the canagliflozin arm in comparison to placebo (43.2\% vs. $61.2 \%)$. These effects were also consistent for the components of ESRD (20.4\% vs. 29.4\%), and doubling of the serum creatinine level $(20.7 \%$ vs. $33.8 \%)$. Other events including reduced eGFR $\left(<15 \mathrm{~mL} / \mathrm{min} / 1.73 \mathrm{~m}^{2}\right)$, dialysis or kidney transplant, and renal mortality were lower in the canagliflozin, but the clinical significance remains questionable as the trial did not report their $\mathrm{P}$ values. The secondary outcomes consisted of cardiovascular death or HF hospitalization (31.5\% vs. $45.4 \%)$, a composite of cardiovascular death, MI or stroke (38.7\% vs. $48.7 \%)$, HF hospitalization ( $15.7 \%$ vs. $25.3 \%$ ), a composite of ESRD, doubling of serum creatinine level, or renal death (27\% vs. 40.4$)$, cardiovascular death (19\% vs. $24.4 \%)$, and finally a composite of death from cardiovascular causes, MI, stroke, HF hospitalization or unstable angina hospitalization (49.4\% vs. $66.9 \%)$. There were less significantly fewer events in the canagliflozin group for most of the secondary outcomes; except for the cardiovascular death (not statistically significant), and both any-cause death and the composite of cardiovascular death, MI, stroke, HF hospitalization, or unstable angina hospitalization ( $\mathrm{P}$-value was not reported). The exploratory outcome consisted of dialysis, kidney transplantation, or renal death, and the study showed lower events in the canagliflozin group; however, the P-value was not reported ( $13.6 \%$ vs. $18.6 \%$ events/1,000 patient-years, HR: $0.72,95 \%$ CI: 0.54 - 0.97). Adverse events that were more common in the canagliflozin group include diabetic ketoacidosis (DKA). However, the study reported no significant difference in the risk of amputation and bone fractures. The study concluded that canagliflozin lowered the risk of cardiovascular events and kidney failure. However, the study was criticized primarily because of its early termination; secondly, because of excluding patients with very advanced kidney disease; third, the decrease in eGFR (about $6 \mathrm{~mL} / \mathrm{min} / 1.73 \mathrm{~m}^{2}$ ) that was seen initially after starting the therapy, and finally, because they did not use statins universally in this trial as they prevent cardiovascular disease and may reduce CKD progression.

\section{DECLARE-TIMI 58}

The DECLARE-TIMI 58 trial published in 2019 [22] was con- 
ducted among diabetic patients ( $\geq 40$ years) with cardiovascular risks to investigate whether administering a daily dose of dapagliflozin reduces the risk of MACE. The trial randomized 17,160 patients to a daily dose of dapagliflozin $10 \mathrm{mg}$ daily $(\mathrm{n}=8,852)$, and placebo $(\mathrm{n}=8,548)$. The primary outcomes were MACE for safety, with MACE and cardiovascular death or HF hospitalization for efficacy. Dapagliflozin met the noninferiority criteria; however, it did not result in a lower rate of MACE compared to placebo ( $8.8 \%$ vs. $9.4 \%$ events $/ 1,000$ patient-years, HR: 0.93, 95\% CI: $0.84-1.03$; P = 0.17). But for efficacy, dapagliflozin lowered the rates of cardiovascular death or HF hospitalization compared to placebo (4.9\% vs. $5.8 \%)$. It is worth noting that the lower rate of the efficacy outcome was due to a lower rate of HF. The secondary outcomes for efficacy were renal composite outcome (sustained decrease of $40 \%$ or more in eGFR to less than $60 \mathrm{~mL} / \mathrm{min} / 1.73$ $\mathrm{m}^{2}$, new ESRD, or death from renal or cardiovascular causes), and any-cause death. The renal composite efficacy outcome occurred less frequently in the dapagliflozin group $(4.3 \% \mathrm{vs}$. $5.6 \%$ ). Also, any-cause death occurred less frequently in the dapagliflozin group but was clinically insignificant $(6.2 \%$ vs. $6.6 \%$ events $/ 1,000$ patient-years, HR: $0.76,95 \%$ CI: 0.82 1.04). Adverse events included genital infections and DKA. The study concluded that adding dapagliflozin led to significantly lower rates of cardiovascular death or HF hospitalization reflecting a lower rate of $\mathrm{HF}$ hospitalization, but did not result in a significant change in MACE. However, the study was criticized due to a potential bias (early discontinuation), a potential conflict of interest (funded by the manufacturer of dapagliflozin), and because it did not meet its primary outcome of MACE improvement. Also, the addition of HF hospitalization might have been an afterthought to improve the trial outcomes. Moreover, using other glucose-lowering drugs than SGLT2 $i$ in the trial might have impacted the results since this was not constant throughout the study.

\section{VERTIS-CV}

The VERTIS-CV trial published in 2020 [23] was conducted among diabetic patients ( $\geq 40$ years) with ASCVD to assess ertugliflozin cardiovascular safety. A total of 8,238 patients were randomized to ertugliflozin $5 \mathrm{mg}(\mathrm{n}=2,752)$, ertugliflozin 15 $\mathrm{mg}(\mathrm{n}=2,747)$, and placebo $(\mathrm{n}=2,747)$. The primary outcome for the study was MACE. It did not meet the statistical significance in ertugliflozin group in comparison to placebo $(3.9 \%$ vs. $4 \%$ events $/ 1,000$ patient-years, HR: $0.97,95 \%$ CI: 0.85 $1.11 ; \mathrm{P}<0.001$ for non-inferiority), even with further analyses of the subgroups. The secondary outcomes were a composite of death from cardiovascular causes or HF hospitalization $(2.3 \%$ vs. $2.7 \%)$, death from cardiovascular causes $(1.8 \%$ vs. $1.9 \%)$, and a composite death from renal causes, renal replacement therapy, or doubling of serum creatinine level (0.9\% vs. $1.2 \%)$, which all were statistically insignificant. However, ertugliflozin showed statistically significant reduction in HF hospitalization compared to placebo $(0.7 \%$ vs. $1.1 \%$ events $/ 1,000$ patient-years, HR: 0.7, 95\% CI: 0.54 - 0.90; P=0.006). Adverse events reported in the trial were genital infections and urinary tract infection (UTI) (statistically significant), with more DKA and amputation events in the ertugliflozin arms. The study concluded that ertugliflozin was non-inferior to placebo with respect to MACE among patients with T2DM and ASCVD. However, the study was criticized because it fell outside its primary and secondary end-points.

\section{Large Randomized Trials in Patients With HF}

\section{EMPA-HEART}

In 2019, Verma et al published the EMPA-HEART trial [24]. The aim of this study was to evaluate the impact of empagliflozin on cardiac function, structure and cardiac biomarkers in patients with T2DM with or without a history of HF. In this randomized, parallel assignment, double masked trial, a total of 97 enrollees were randomized to receive a daily dose of either empagliflozin $10 \mathrm{mg}(\mathrm{n}=49)$ or placebo $(\mathrm{n}=48)$. These patients were then followed for 6 months. The primary outcomes consisted of the delta in left ventricular mass index as noted on cardiac magnetic resonance imaging (MRI) in patients receiving empagliflozin vs. placebo of $-2.6 \mathrm{~g} / \mathrm{m}^{2}$ in empagliflozin group vs. $-0.0 .01 \mathrm{~g} / \mathrm{m}^{2}$ in the placebo group with $\mathrm{P}$ value of 0.01 . Of note, the most significant benefit was seen amongst patients with a left ventricular mass index greater than $60 \mathrm{~g} /$ $\mathrm{m}^{2}$. The secondary outcomes were as follows: change in ejection fraction $0.72 \%$ vs. $-1.0 \%(\mathrm{P}=0.08)$, change in left ventricular end-systolic volume index -1.0 vs. $0.04 \mathrm{~mL} / \mathrm{m}^{2}(\mathrm{P}=0.36)$, change in hematocrit $2.4 \%$ vs. $0.4 \%(\mathrm{P}=0.006)$, and change in systolic blood pressure -7.9 vs. $-0.7 \mathrm{~mm} \mathrm{Hg}(\mathrm{P}=0.003)$. The aforementioned results show that empagliflozin did in fact have a beneficial effect on cardiac remodeling; however, it is important to note that this benefit could not be ascertained in patients with a reduced ejection fraction as only $6 \%$ of patients in this study had a prior known ejection fraction.

\section{DEFINE-HF}

Nassif et al also published a trial in 2019 called the DEFINEHF trial to evaluate the effects of dapagliflozin in patients with reduced ejection fraction with or without T2DM [25]. This trial was a multi-center, investigator-initiated, randomized trial which included patients with an ejection fraction of $\leq 40 \%$, NYHA class II-III, eGFR $\geq 30 \mathrm{~mL} / \mathrm{min} / 1.73 \mathrm{~m}^{2}$ and elevated B-type natriuretic peptide (BNP). The study randomized 263 patients to receive $10 \mathrm{mg}$ of dapagliflozin or placebo daily for a total of 12 weeks. The primary outcomes of this trial were as follows: difference in N-terminal pro-BNP (NT-proBNP) at 6 and 12 weeks in dapagliflozin vs. placebo $(1,133 \mathrm{pg} / \mathrm{dL}$ vs. $1,191 \mathrm{pg} / \mathrm{dL}, \mathrm{P}=0.43)$, improvement of $\geq 5$ points in the Kansas City Cardiomyopathy Questionnaire (KCCQ) or a $\geq$ $20 \%$ decrease in NT-proBNP, $61.5 \%$ of patients treated with dapagliflozin met the end-point as compared to $50.4 \%$ treated with placebo $(\mathrm{P}=0.039)$, these results were similar across groups regardless of T2DM. Results of this trial highlight that although there was no statistically significant improvement in the mean natriuretic peptide levels, there was an increase in the 
number of patients experiencing improvement in health status regardless of diabetic status.

\section{PRESERVED-HF}

To assess the benefits of dapagliflozin in patients with HF$\mathrm{pEF}$, the PRESERVED-HF trial is currently underway [26] to assess the effects of dapagliflozin in patients with preserved ejection fraction pertinent to cardiac biomarkers, symptoms and impact on the overall quality of life. It is designed as a 12week, randomized, double-blinded, placebo-controlled trial in which patients received either $10 \mathrm{mg}$ dapagliflozin or placebo. Primary end-points included symptomatic and physical limitations as measured by the KCCQ. Secondary outcomes included change in NT-proBNP, BNP, 6-min walk test, hemoglobin A1c, weight, and systolic blood pressures at 6 and 12 weeks. The results of this study were estimated to be complete in June 2021 and will provide further direction as to the potential use of dapagliflozin in patients with HFpEF.

\section{DAPA-HF}

DAPA-HF was conducted in patients with HFrEF to evaluate the effect of a daily dose of dapagliflozin $10 \mathrm{mg}$ in comparison to placebo, in addition to standard care [26]. The study randomized 4,744 HFrEF patients and an eGFR $\geq 30 \mathrm{~mL} / \mathrm{min} / 1.73$ $\mathrm{m}^{2}$ to dapagliflozin $10 \mathrm{mg}$ daily $(\mathrm{n}=2,373)$ or a placebo $(\mathrm{n}=$ 2,371 ), irrespective of T2DM status for 18.2 months followup. The mean age was 66 years, $24 \%$ were females and $42 \%$ were diabetic. The primary outcome consisted of deaths from cardiovascular causes, HF hospitalization, or urgent HF visit, which were significantly lower in dapagliflozin group $(16.3 \%$ vs. $21.2 \%$ ) with $\mathrm{P}<0.001$. The secondary outcomes were cardiovascular death, HF hospitalization, and worsening of renal function, which all were lower in dapagliflozin group. In conclusion, this trial showed that dapagliflozin lowered the risk of death and HF hospitalization in patients with HFrEF, compared to placebo, regardless of the presence or absence of diabetes.

\section{EMPEROR-REDUCED}

The aim of the EMPEROR-REDUCED trial was to determine the impact of an SGLT2 inhibitor called empagliflozin on lowering the chances of hospitalization for HFrEF in patients with or without diabetes [27]. The study randomized 3,730 patients with class II, III, or IV HFrEF to a daily dose of empagliflozin $10 \mathrm{mg}$ or placebo. The composite primary outcome consisted of cardiovascular death or HF hospitalization. The primary outcome was significantly less in the empagliflozin group (19.4\% vs. $24.7 \%$ with $\mathrm{P}$ value $<0.001)$. Secondary outcomes consisted of total number of HF hospitalization, which was also significantly lower in the empagliflozin group (388 events vs. 553 events). Also, the rate of eGFR decline was significantly lower in the empagliflozin group during the study time. Adverse events included genital tract infection. Overall, the study concluded that empagliflozin was associated with a lower risk of cardiovascular death and HF hospitalization with a slower progressive decline in renal function in patients with HFrEF, regardless of T2DM status.

\section{EMPEROR-PRESERVED}

The aim of the EMPEROR-PRESERVED was to evaluate the effects of the SGLT2 inhibitor empagliflozin in patients with HFpEF, regardless of T2DM status [28]. The trial enrolled 5,750 patients with HFpEF with or without T2DM. Patients were randomized to a daily dose of empagliflozin $10 \mathrm{mg}$ or a placebo. The primary was the time-to-first-event analysis of the combined risk for cardiovascular death and HF hospitalization. Secondary outcomes were HF hospitalization, reduction of eGFR, dialysis or renal transplant occurrence, time to cardiovascular death, time to all-cause mortality, and time to onset of T2DM. The results of this study were estimated to be complete in April 2021 and will assess the impact of empagliflozin use in patients with HFpEF (with or without DM) on morbidity and mortality.

\section{EMBRACE-HF}

The EMBRACE-HF trial is a 12-week randomized trial that was conducted to explore the effects of a daily dose of empagliflozin $10 \mathrm{mg}$ on pulmonary artery (PA) pressures in patients with $\mathrm{HF}$, regardless of EF status, who already have a CardioMEMs device implanted for non-study-related clinical reasons [29]. The study randomized 60 patients. The primary end-point was the delta change in pulmonary artery pressure from baseline to end of treatment. Secondary endpoints were the delta change from baseline in pulmonary artery diastolic pressure (PADP) at each interim time point, delta change from baseline to follow-up in HF-related quality of life between empagliflozin and placebo, using KCCQ score, change in 6-min walk test from baseline to follow-up (defined as average of measurements at 6 and 12 weeks) between empagliflozin and placebo. Empagliflozin significantly reduced PADP, with effects beginning at week 1, and amplified over time; average PADP (weeks 8 - 12) was 1.5 mm Hg lower (95\% CI: 0.2 - 2.8; $\mathrm{P}=0.02)$; and at week 12 , PADP was $1.7 \mathrm{~mm} \mathrm{Hg}$ lower $(95 \%$ CI: 0.3 - 3.2; P=0.02) with empagliflozin vs. placebo. Results were consistent for PA systolic and PA mean pressures. There was no difference in mean loop diuretic management (daily furosemide equivalents) between treatment groups. No significant differences between treatment groups were observed in $\mathrm{KCCQ}$, natriuretic peptide levels and 6-min walking distance. Although empagliflozin showed significant reduction in PADP $(\mathrm{P}=0.02)$, the study does not reflect mortality or morbidity rates. Furthermore, only 60 participants were included in the study which potentially reflects low study power.

\section{REFORM}

The REFORM trial published in 2020 [30] was conducted 
amongst 56 T2DM patients with HF and LV systolic dysfunction. Patients were randomized to a daily dose of dapagliflozin $10 \mathrm{mg}$ daily $(\mathrm{n}=28)$ versus placebo for 1 year $(\mathrm{n}=28)$. The study was designed specifically to test the safety and efficacy of the SLGT2 inhibitor, dapagliflozin, on diabetic patients with known HF. The primary end-point was the difference in LV end-systolic volume (LVESV) using cardiac MRI. After 1 year of medication therapy, the primary end-point was not met. The secondary outcomes were investigated: changes in LV mass index $\left(2.5 \mathrm{~g} / \mathrm{m}^{2}(-3.95\right.$ to 8.95$\left.), \mathrm{P}=0.440\right)$, left ventricular ejection fraction $(0.96 \%(-3.32$ to 4.69$), \mathrm{P}=0.732), \mathrm{LV}$ stroke volume $(1.86 \mathrm{~mL}(-1.52$ to 5.24$), \mathrm{P}=0.273)$, indexed left atrial volume $\left(-2.6 \mathrm{~mL} / \mathrm{m}^{2}(-9.97\right.$ to 4.48$\left.), \mathrm{P}=0.464\right)$, weight $(-1.36 \mathrm{~kg}(-4.14$ to 1.42$), \mathrm{P}=0.329), \mathrm{HbAlc}(-1.49 \mathrm{mmol} / \mathrm{mol}$ (-6.95 to 3.97), $\mathrm{P}=0.586)$ and GFR (1.96 (-4.78 to 8.70), $\mathrm{P}=$ $0.563)$. However, they remained statistically insignificant. The secondary outcomes that were statistically significant included patients on dapagliflozin who required less loop diuretic therapy. Further evidence demonstrated dapagliflozin significantly reduced diastolic blood pressure, without a difference in systolic BP or heart rate. Dapagliflozin significantly increased hemoglobin, hematocrit, and fasting ketone bodies. Adverse events included decline in renal function, which was transient and resolved after reduction of loop diuretic dose without dapagliflozin dose change. Overall, the study concluded that dapagliflozin therapy did not reverse LV remodeling after 1 year. However, there was a significant difference in loop diuretic requirements, systolic blood pressure, hematocrit, and fasting ketone levels between groups. Limitations of the study include a patient population with mild severity of HF on a modest dose of diuretic (max. $80 \mathrm{mg}$ or equivalent), thus only evaluating the function of dapagliflozin in patients with lesser severity of the disease. Additionally, the small sample size of the trial does not allow sufficient conclusions to be drawn and the trial should have been done on a larger scale to improve the power of the study. Lastly, loop diuretic dose adjustments may have led to ventricular changes, resulting in confounding results.

\section{EMPA}

The EMPA trial is a randomized trial with a crossover design published in 2020 [31]. It was conducted amongst 20 people with HF and T2DM. Patients were randomized to a daily dose of empagliflozin $10 \mathrm{mg}$ or placebo for 14 days followed by a 2-week washout period and crossover to 14 days of treatment with an alternate agent. The goal of the study was to identify the acute and intermediate effects of SGLT2 inhibitors on sodium elimination, patient's volume status, and neurohormonal activation in HF patients within 14 days of therapy. The primary outcome was to determine if empagliflozin would improve the loop diuretic's natriuretic effect. Empagliflozin was associated with a statistically significant 27 -fold increase in glycosuria and natruresis $(1.2 \pm 0.7 \%$ vs. $0.7 \pm 0.4 \%)$, with a synergistic effect in combination with bumetanide $(5.8 \pm 2.5 \%$ vs. $3.9 \pm 1.9 \%$ ). Secondary outcomes included determining the effect of SGLT2 inhibition on blood volume after 14 days. At this period mark, there was a statistically significant reduction in blood volume and plasma volume. However, there was no significant RAAS or sympathetic nervous system activation with empagliflozin. Additionally, there was no serious electrolyte abnormality observed amongst the two groups. There were no adverse events documented or reported in the trial. Overall, the study concluded that empagliflozin had a statistically and clinically significant natriuretic effect when used as monotherapy and a synergistic effect when combined with a loop diuretic. Limitations of the trial included its small sample size and mechanistic design, which consequently does not allow further information such as adverse events to be observed. Additionally, the generalizability of the study was questioned as only patients with stable HF and T2DM were studied.

\section{SOLOIST-WHF}

The SOLOIST-WHF trial (Effect of Sotagliflozin on Cardiovascular Events in Patients With Type 2 Diabetes Post Worsening Heart Failure published in 2020 [32] was conducted amongst 1,222 patients ( $\geq 18$ years) with T2DM who were recently hospitalized for HF exacerbation. The trial was conducted to investigate sotagliflozin safety and efficacy on reducing the cardiovascular events in T2DM patients with recent HF admission. The study randomized 1,222 patients to either sotagliflozin group $(n=608)$ or placebo $(n=614)$ group. The primary outcome consisted of the combined death of cardiovascular causes, HF hospitalizations, and urgent HF visits. Both primary end-point events were significantly lower in the sotagliflozin group (51.0 vs. 76.3) than the placebo group. The secondary outcomes for the study included the total cardiovascular death and HF hospitalizations, first cardiovascular death and HF hospitalization, cardiovascular death, change in KCCQ, and change in eGFR. There was a significant difference in the secondary outcome between the sotagliflozin group and the placebo group, except for cardiovascular death and changes in eGFR. Adverse events reported in the study were diarrhea, genital fungal infections, and severe hypoglycemia. The study concluded that T2DM patients with recent worsening HF who received sotagliflozin therapy right before or shortly after discharge events had reduced risks of a composite of cardiovascular death, HF hospitalization, or urgent HF visit for HF vs. placebo. The trial was criticized for its shortened duration and loss of power as it was ended because of COVID-19 pandemic.

\section{Conclusions}

HF is a leading cause of morbidity and mortality. Based on the results of many clinical trials, SGLT2 inhibitors have not only shown substantial cardiovascular benefits (reduction in the risk of HFrEF hospitalizations or composite cardiovascular deaths), but also reduction of kidney diseases regardless of diabetes status (Table 1). So far, no effective treatment improves the morbidity and mortality in patients with HFpEF, but, with the promising result from EMPRISE trial, we are optimistic that the ongoing trials EMPERIAL-PRESERVED and EMPERIAL-REDUCED on exercise ability including 6-min walking 
Table 1. A Summary for SGLT2 Inhibitors Clinical Trials Including the Studied Drug, Primary Outcome, NNT for the Primary Outcome, and the Reported Adverse Events in Each Trial

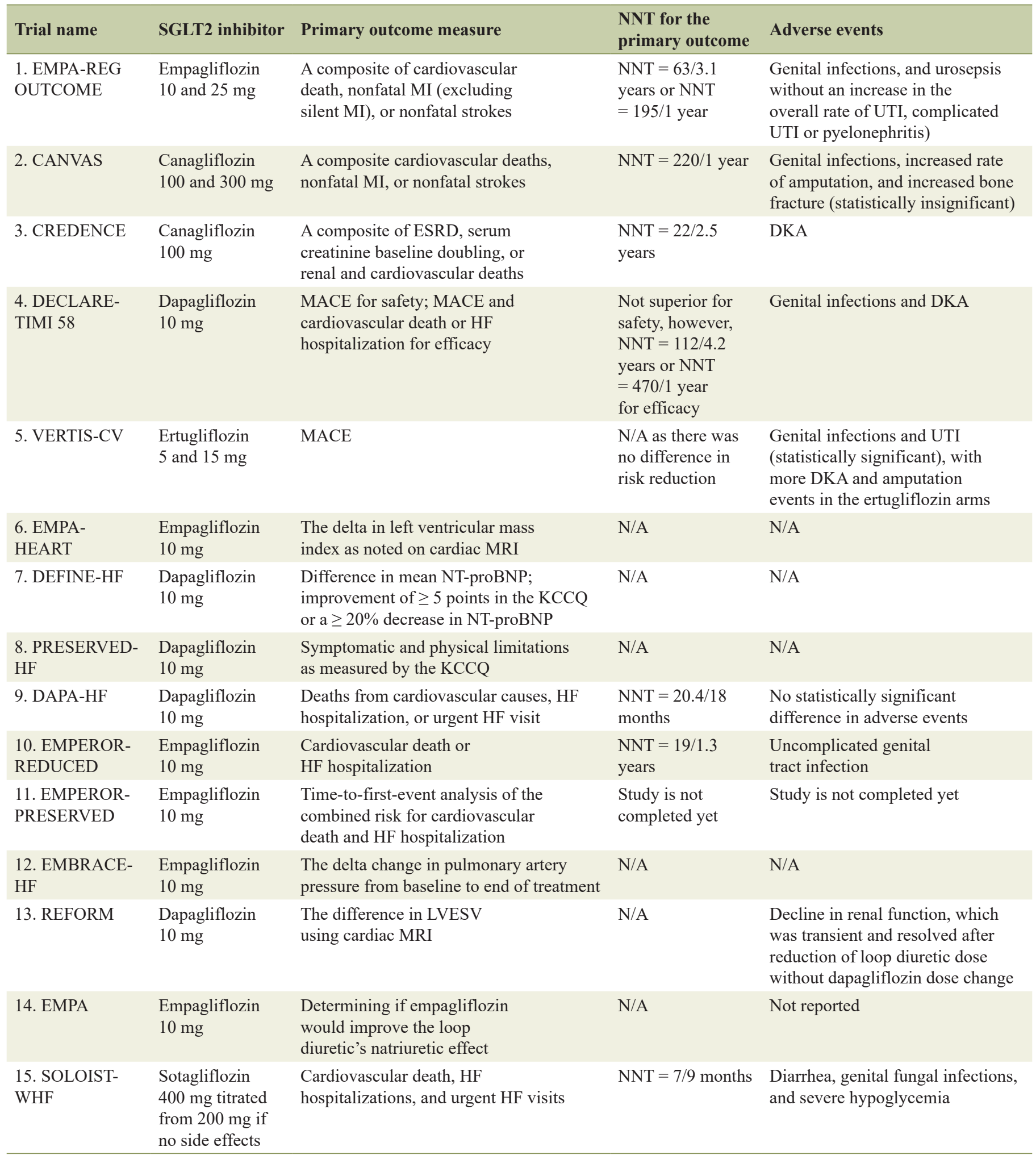

SGLT2: sodium-glucose co-transporter-2; NNT: number needed to treat; MI: myocardial infarction; UTI: urinary tract infection; ESRD: end-stage renal disease; DKA: diabetic ketoacidosis; MACE: major adverse cardiovascular event; HF: heart failure; MRI: magnetic resonance imaging; NT-proBNP: N-terminal pro-B-type natriuretic peptide; KCCQ: Kansas City Cardiomyopathy Questionnaire; LVESV: left ventricular end-systolic volume. 
test in each respective HF phenotype will further guide broader use of SGLT2 $\mathrm{i}$ in HF treatment.

\section{Acknowledgments}

None to declare.

\section{Financial Disclosure}

None to declare.

\section{Conflict of Interest}

None to declare.

\section{Author Contributions}

Randa Abdelmasih: abstract and introduction, Ramy Abdelmaseih: discussion, studies 1-3, Ravi Thakker: studies 4-6, Mohammed Faluk: studies 7-9, Arroj Ali: studies 10-12, Mrhaf Alsamman: studies 13-14, Syed Mustajab Hasan: study 15, and conclusion. Authors made substantial contributions to conception. Authors participated in drafting the article and revising it critically for important intellectual content. Authors gave final approval of the version to be submitted and any revised version.

\section{Data Availability}

The authors declare that data supporting the findings of this study are available within the article.

\section{References}

1. Virani SS, Alonso A, Benjamin EJ, Bittencourt MS, Callaway CW, Carson AP, Chamberlain AM, et al. Heart disease and stroke statistics-2020 update: a report from the American Heart Association. Circulation. 2020;141(9): e139-e596.

2. Lloyd-Jones DM, Larson MG, Leip EP, Beiser A, D'Agostino RB, Kannel WB, Murabito JM, et al. Lifetime risk for developing congestive heart failure: the Framingham Heart Study. Circulation. 2002;106(24):3068-3072.

3. Jencks SF, Williams MV, Coleman EA. Rehospitalizations among patients in the Medicare fee-for-service program. N Engl J Med. 2009;360(14):1418-1428.

4. Urbich M, Globe G, Pantiri K, Heisen M, Bennison C, Wirtz HS, Di Tanna GL. A systematic review of medical costs associated with heart failure in the USA (20142020). Pharmacoeconomics. 2020;38(11):1219-1236.

5. Ho KK, Pinsky JL, Kannel WB, Levy D. The epidemiology of heart failure: the Framingham Study. J Am Coll
Cardiol. 1993;22(4 Suppl A):6A-13A.

6. American Heart Association. Classes of heart failure. Available at: http://www.heart.org/HEARTORG/Conditions/HeartFailure/AboutHeartFailure/Classes-of-HeartFailure UCM 306328 Article.jsp\#.WUcGf-vyuHs. Updated: May 8, 2017; Accessed: June 18, 2017.

7. Writing Committee Members, Yancy CW, Jessup M, Bozkurt B, Butler J, Casey DE, Jr., Drazner MH, et al. 2013 ACCF/AHA guideline for the management of heart failure: a report of the American College of Cardiology Foundation/American Heart Association Task Force on practice guidelines. Circulation. 2013;128(16):e240-327.

8. Vasan RS, Xanthakis V, Lyass A, Andersson C, Tsao C, Cheng S, Aragam J, et al. Epidemiology of left ventricular systolic dysfunction and heart failure in the Framingham study: an echocardiographic study over 3 decades. JACC Cardiovasc Imaging. 2018;11(1):1-11.

9. Bytyci I, Bajraktari G. Mortality in heart failure patients. Anatol J Cardiol. 2015;15(1):63-68.

10. Garber AJ, Abrahamson MJ, Barzilay JI, Blonde L, Bloomgarden ZT, Bush MA, Dagogo-Jack S, et al. Consensus statement by the american association of clinical endocrinologists and American College of Endocrinology on the comprehensive type 2 diabetes management algorithm - 2019 executive summary. Endocr Pract. 2019;25(1):69-100.

11. Goldenberg RM, Berard LD, Cheng AYY, Gilbert JD, Verma S, Woo VC, Yale JF. SGLT2 inhibitor-associated diabetic ketoacidosis: clinical review and recommendations for prevention and diagnosis. Clin Ther. 2016;38(12): 2654-2664 e2651.

12. Cherney DZ, Perkins BA, Soleymanlou N, Maione M, Lai V, Lee A, Fagan NM, et al. Renal hemodynamic effect of sodium-glucose cotransporter 2 inhibition in patients with type 1 diabetes mellitus. Circulation. 2014;129(5):587597.

13. Tanaka H, Takano K, Iijima H, Kubo H, Maruyama N, Hashimoto T, Arakawa K, et al. Factors affecting canagliflozin-induced transient urine volume increase in patients with type 2 diabetes mellitus. Adv Ther. 2017;34(2):436451.

14. Lam CSP, Chandramouli C, Ahooja V, Verma S. SGLT2 inhibitors in heart failure: current management, unmet needs, and therapeutic prospects. J Am Heart Assoc. 2019;8(20):e013389.

15. Verma S. Potential Mechanisms of Sodium-Glucose CoTransporter 2 Inhibitor-Related Cardiovascular Benefits. Am J Cardiol. 2019;124(Suppl 1):S36-S44.

16. Zelniker TA, Wiviott SD, Raz I, Im K, Goodrich EL, Bonaca MP, Mosenzon O, et al. SGLT2 inhibitors for primary and secondary prevention of cardiovascular and renal outcomes in type 2 diabetes: a systematic review and meta-analysis of cardiovascular outcome trials. Lancet. 2019;393(10166):31-39.

17. Udell JA, Yuan Z, Rush T, Sicignano NM, Galitz M, Rosenthal N. Cardiovascular outcomes and risks after initiation of a sodium glucose cotransporter 2 inhibitor: results from the EASEL population-based cohort study (Evidence for cardiovascular outcomes with sodium glu- 
cose cotransporter 2 inhibitors in the real world). Circulation. 2018;137(14):1450-1459.

18. Filion KB, Lix LM, Yu OH, Dell'Aniello S, Douros A, Shah BR, St-Jean A, et al. Sodium glucose cotransporter 2 inhibitors and risk of major adverse cardiovascular events: multi-database retrospective cohort study. BMJ. 2020;370:m3342.

19. Zinman B, Wanner C, Lachin JM, Fitchett D, Bluhmki E, Hantel S, Mattheus M, et al. Empagliflozin, cardiovascular outcomes, and mortality in type 2 diabetes. N Engl J Med. 2015;373(22):2117-2128.

20. Neal B, Perkovic V, Mahaffey KW, de Zeeuw D, Fulcher G, Erondu N, Shaw W, et al. Canagliflozin and cardiovascular and renal events in type 2 diabetes. N Engl J Med. 2017;377(7):644-657.

21. Perkovic V, Jardine MJ, Neal B, Bompoint S, Heerspink HJL, Charytan DM, Edwards R, et al. Canagliflozin and renal outcomes in type 2 diabetes and nephropathy. N Engl J Med. 2019;380(24):2295-2306.

22. Wiviott SD, Raz I, Bonaca MP, Mosenzon O, Kato ET, Cahn A, Silverman MG, et al. Dapagliflozin and cardiovascular outcomes in type 2 diabetes. N Engl J Med. 2019;380(4):347-357.

23. Cosentino F, Cannon CP, Cherney DZI, Masiukiewicz U, Pratley R, Dagogo-Jack S, Frederich R, et al. Efficacy of ertugliflozin on heart failure-related events in patients with type 2 diabetes mellitus and established atherosclerotic cardiovascular disease: results of the VERTIS CV trial. Circulation. 2020;142(23):2205-2215.

24. Bami K, Gandhi S, Leong-Poi H, Yan A, Ho E, Zahrani M, Connelly K. Effects of empagliflozin on cardiac function in patients with type 2 diabetes mellitus: Echocardiographic substudy of the EMPA-HEART cardiolink-6 trial. Eur Heart J. 2019;40(Supplement_1):1500.

25. Kosiborod M, Nassif M, Windsor S, Tang F, Khariton Y, Austin B, Scirica B. Effects of dapagliflozin on biomarkers, symptoms and functional status in patients with heart failure with reduced ejection fraction with and without diabetes - the Define-HF trial. Journal of Cardiac Failure. 2019;25(11):937-938.

26. McMurray JJV, Solomon SD, Inzucchi SE, Kober L, Kosiborod MN, Martinez FA, Ponikowski P, et al. Dapagliflozin in Patients with Heart Failure and Reduced Ejection Fraction. N Engl J Med. 2019;381(21):1995-2008.

27. Packer M, Anker SD, Butler J, Filippatos G, Pocock SJ, Carson P, Januzzi J, et al. Cardiovascular and renal outcomes with empagliflozin in heart failure. N Engl J Med. 2020;383(15):1413-1424.

28. Anker SD, Butler J, Filippatos GS, Jamal W, Salsali A, Schnee J, Kimura K, et al. Evaluation of the effects of sodium-glucose co-transporter 2 inhibition with empagliflozin on morbidity and mortality in patients with chronic heart failure and a preserved ejection fraction: rationale for and design of the EMPEROR-Preserved Trial. Eur J Heart Fail. 2019;21(10):1279-1287.

29. Nassif ME, Qintar M, Windsor SL, Jermyn R, Shavelle DM, Tang F, Lamba S, et al. Empagliflozin Effects on Pulmonary Artery Pressure in Patients With Heart Failure: Results From the EMBRACE-HF Trial. Circulation. 2021;143(17):1673-1686.

30. Singh JS, Fathi A, Vickneson K, Mordi I, Mohan M, Houston JG, Pearson ER, et al. Research into the effect Of SGLT2 inhibition on left ventricular remodelling in patients with heart failure and diabetes mellitus (REFORM) trial rationale and design. Cardiovasc Diabetol. 2016;15:97.

31. Griffin M, Rao VS, Ivey-Miranda J, Fleming J, Mahoney D, Maulion C, Suda N, et al. Empagliflozin in heart failure: diuretic and cardiorenal effects. Circulation. 2020;142(11):1028-1039.

32. Bhatt DL, Szarek M, Steg PG, Cannon CP, Leiter LA, McGuire DK, Lewis JB, et al. Sotagliflozin in patients with diabetes and recent worsening heart failure. N Engl J Med. 2021;384(2):117-128. 